\title{
Impact of compact fluorescent lamps on energy transmission losses and power quality
}

\author{
Gorazd Štumberger ${ }^{1}$, Klemen Deželak ${ }^{1}$, Sebastijan Seme ${ }^{1}$, Miran Rošer ${ }^{2}$, Viktor Tajnšek ${ }^{2}$ \\ ${ }^{1}$ University of Maribor \\ Faculty of Electrical Engineering and Computer Science \\ Smetanova 17, 2000 Maribor, Slovenia \\ Phone:+386 2220 7075, Fax: +386 22207272 , \\ e-mail: gorazd.stumberger@uni-mb.si, klemen.dezelak@uni-mb.si, sebastijan.seme@uni-mb.si \\ ${ }^{2}$ Elektro Celje d.d. \\ Vrunčeva 2a, 3000 Celje, Slovenia \\ Phone: +386 34201 1000, Fax: +386 35485023 \\ e-mail: miran.roser@elektro-celje.si, viki.tajnsek@elektro-celje.si
}

\begin{abstract}
The compact fluorescent lamps (CFLs), called also saving bulbs, produce the same luminous flux as incandescent lamps (normal bulbs) (ILs), but require only 20 to 25 percents of the power required by the ILs. However, it is often neglected that CLFs behave as nonlinear loads, causing harmonic distortion of currents and voltages and additional losses related with the energy transmission. In the case study, this work evaluates the impact of CFLs on the power quality and increase of losses related with the energy transmission.
\end{abstract}

Key words: compact fluorescent lamps, power quality, total harmonic distortion, energy transmission losses, orthogonal decomposition of currents

\section{Introduction}

The energy shortage on one hand and global warming on the other hand, force us to reduce energy consumption and emissions of greenhouse gases. In the field of lighting the energy consumption can be reduced using compact fluorescent lamps (CFLs), called also saving bulbs, or using light emission diodes (LEDs). At present time, usage of the last ones is more or less limited to the automotive industry and special applications, but they are not widely spread in households and offices where usage of the CFLs is increasing. The CFLs consume approximately 20 to 25 percents of the energy consumed by the incandescent lamps (ILs) (normal bulbs) to produce the same luminous flux. From the viewpoint of energy saving the advantages of CFLs are clear. However, the CFLs have also drawbacks, which are often neglected. They represent nonlinear loads which increase total harmonic distortion of currents and voltages and increase losses related with the energy transmission. In the case study, the impact of CFLs on losses related with the energy transmission and distortion of power quality are discussed in this work. In order to perform the analysis orthogonal decomposition of currents [1]-[4] in the time domain and Fourier analysis are applied.

\section{Orthogonal decomposition of currents in time domain}

Let $u_{a}(t), u_{b}(t)$ and $u_{c}(t)$ be the voltages and $i_{a}(t), i_{b}(t)$ and $i_{c}(t)$ be the currents of a three-phase system observed over a selected time interval $t \in[0, T]$, where they can be treated as continuous functions. In order to determine current necessary for energy transmission from the source to the load, presented in the form of active power $P$, current vector $\mathbf{i}$ and voltage vector $\mathbf{u}$ are introduced by (1).

$$
\mathbf{i}=\left[\begin{array}{c}
i_{a}(t) \\
i_{b}(t) \\
i_{c}(t)
\end{array}\right], \quad \mathbf{u}=\left[\begin{array}{l}
u_{a}(t) \\
u_{b}(t) \\
u_{c}(t)
\end{array}\right]
$$

The inner product of both vectors $(\mathbf{u}, \mathbf{i})$ is introduced by (2):

$$
\begin{aligned}
(\mathbf{u}, \mathbf{i}) & =\frac{1}{T} \int_{0}^{T} \mathbf{u}^{T} \mathbf{i} d t \\
& =\frac{1}{T} \int_{0}^{T}\left(u_{a}(t) i_{a}(t)+u_{b}(t) i_{b}(t)+u_{c}(t) i_{c}(t)\right) d t \\
& =P_{a}+P_{b}+P_{c}=P
\end{aligned}
$$

where $P_{a}, P_{b}$, and $P_{c}$ are active powers of individual phases wile $P$ is the active power for the entire three- 
phase system. In the similar way squares of the current vector norm $\|\mathbf{i}\|^{2}$ and voltage vector norm $\|\mathbf{u}\|^{2}$ are introduced by (3) and (4), respectively:

$$
\begin{aligned}
\|\mathbf{i}\|^{2}=(\mathbf{i}, \mathbf{i})= & \frac{1}{T} \int_{0}^{T} \mathbf{i}^{T} \mathbf{i} d t \\
= & \frac{1}{T} \int_{0}^{T}\left(i_{a}^{2}(t)+i_{b}^{2}(t)+i_{c}^{2}(t)\right) d t \\
& =I_{a}^{2}+I_{b}^{2}+I_{c}^{2} \\
\|\mathbf{u}\|^{2}=(\mathbf{u}, \mathbf{u}) & =\frac{1}{T} \int_{0}^{T} \mathbf{u}^{T} \mathbf{u} d t \\
& =\frac{1}{T} \int_{0}^{T}\left(u_{a}^{2}(t)+u_{b}^{2}(t)+u_{c}^{2}(t)\right) d t \\
& =U_{a}^{2}+U_{b}^{2}+U_{c}^{2}
\end{aligned}
$$

where the squares of RMS values of currents and voltages in individual phases are denoted by $I_{a}{ }^{2}, I_{b}{ }^{2}, I_{c}{ }^{2}$ and $U_{a}^{2}, U_{b}^{2}, U_{c}^{2}$.

The current vector $\mathbf{i}$ (1) can be decomposed into two orthogonal components. The first one denoted with $\mathbf{i}_{\mathrm{u}}$ (5) represents the current vector component aligned with the voltage vector $\mathbf{u}$. The current vector $\mathbf{i}_{\mathrm{u}}$ is indispensable for energy transmission from the source to the load, represented in the form of active power $P$.

$$
\mathbf{i}_{u}=\left[\begin{array}{c}
i_{a u} \\
i_{b u} \\
i_{c u}
\end{array}\right]=\frac{P}{\|\mathbf{u}\|^{2}} \mathbf{u}
$$

The second current vector component denoted with $\mathbf{i}_{\mathrm{uo}}$ (6) is orthogonal to the voltage vector $\mathbf{u}$ and does not contribute to the active power $P$.

$$
\mathbf{i}_{\text {ио }}=\left[\begin{array}{l}
i_{\text {аио }} \\
i_{\text {bио }} \\
i_{\text {сио }}
\end{array}\right]=\mathbf{i}-\mathbf{i}_{u}
$$

The current vector components $\mathbf{i}_{\mathrm{u}}$ and $\mathbf{i}_{\mathrm{uo}}$ are orthogonal, which means that their inner product equals zero (7).

$$
\left(\mathbf{i}_{u}, \mathbf{i}_{\text {uо }}\right)=0
$$

The norms of current vector $\mathbf{i}$ and its orthogonal components $\mathbf{i}_{\mathrm{u}}$ and $\mathbf{i}_{\mathrm{uo}}$ are related by (8).

$$
\|\mathbf{i}\|^{2}=\left\|\mathbf{i}_{u}\right\|^{2}+\left\|\mathbf{i}_{u o}\right\|^{2}
$$

The losses in the electric lines related with energy transmission are function of $\|\mathbf{i}\|^{2}$, while for the active power, which is proportional to the average energy transmission, only current vector component $\mathbf{i}_{\mathrm{u}}$ is indispensable.
The generalized power factor $P F$ can be defined by (9):

$$
P F=\frac{\left\|\mathbf{i}_{u}\right\|}{\|\mathbf{i}\|}=\frac{\left\|\mathbf{i}_{u}\right\|\|\mathbf{u}\|}{\|\mathbf{i}\|\|\mathbf{u}\|}=\frac{|P|}{\|\mathbf{i}\|\|\mathbf{u}\|}
$$

In the case of a single phase system, the definitions for the current vector $\mathbf{i}$ and voltage vector $\mathbf{u}$, given by (1), change to (10), which must be considered also in (2) to (9).

$$
\mathbf{i}=\left[i_{a}(t)\right], \quad \mathbf{u}=\left[u_{a}(t)\right]
$$

\section{Analysis}

In order to evaluate losses related with the energy transmission in the cases of single- and three-phase load composed of CFLs and ILs, the active power $P$, the voltage vector $\mathbf{u}$ and the current vectors $\mathbf{i}, \mathbf{i}_{\mathrm{u}}$ and $\mathbf{i}_{\mathrm{uo}}$ have to be determined. The neutral conductor currents due to the current vectors $\mathbf{i}, \mathbf{i}_{\mathrm{u}}$ and $\mathbf{i}_{\mathrm{uo}}$ are denoted by $i_{n}(11), i_{n u}$ (12) and $i_{\text {пио }}(13)$, respectively.

$$
\begin{aligned}
& i_{n}=i_{a}+i_{b}+i_{c} \\
& i_{\text {пи }}=i_{\text {аи }}+i_{b u}+i_{c u} \\
& i_{\text {пио }}=i_{\text {аио }}+i_{\text {bио }}+i_{\text {сио }}
\end{aligned}
$$

The losses related with the energy transmission appear in the line conductors and in the neutral conductor. The losses $P_{l u}$ (14) appear due to the current vector component $i_{\mathbf{u}}$ and cannot be avoided. The additional losses that could be avoided $P_{\text {luo }}(15)$ are caused by the current vector component $i_{\mathbf{u o}}$. The total losses $P_{l}(16)$ are caused by the current vector $\mathbf{i}$.

$$
\begin{aligned}
& P_{\text {lи }}=R\left\|\mathbf{i}_{u}\right\|^{2}+R_{n}\left\|i_{\text {пи }}\right\|^{2} \\
& P_{\text {lио }}=R\left\|\mathbf{i}_{\text {ио }}\right\|^{2}+R_{n}\left\|i_{\text {nио }}\right\|^{2} \\
& P_{l}=R\|\mathbf{i}\|^{2}+R_{n}\left\|i_{n}\right\|^{2}
\end{aligned}
$$

The resistances of the line and neutral conductors are marked by $R$ and $R_{n}$, respectively. The RMS values of neutral conductor currents $i_{n}, i_{n u}$ and $i_{n u о}$ are marked with $\left\|i_{n}\right\|,\left\|i_{n u}\right\|$ and $\left\|i_{\text {nuo }}\right\|$. The ratio $L F$, given by (17), is defined as the ratio between the losses related with the energy transmission and the load power.

$$
L F=\frac{P_{l}}{P}=\frac{R\|\mathbf{i}\|^{2}+R_{n}\left\|i_{n}\right\|^{2}}{(\mathbf{u}, \mathbf{i})}=\frac{R\|\mathbf{i}\|^{2}+R_{n}\left\|i_{n}\right\|^{2}}{\|\mathbf{u}\|\left\|\mathbf{i}_{u}\right\|}
$$

Considering (9), (17) can be written by (18).

$$
L F=\frac{R\|\mathbf{i}\|^{2}+R_{n}\left\|i_{n}\right\|^{2}}{\|\mathbf{u}\|\|\mathbf{i}\| P F}=\frac{\|\mathbf{i}\|}{\|\mathbf{u}\|} \frac{R}{P F}+\frac{\left\|i_{n}\right\|^{2}}{\|\mathbf{u}\|\|\mathbf{i}\|} \frac{R_{n}}{P F}
$$


The total harmonic distortions of individual line voltages $T H D_{u}$ and line currents $T H D_{i}$ are defined by (19) and (20):

$$
\begin{aligned}
\text {THD}_{u} & =\frac{\sum_{h=2}^{40} U_{h}^{2}}{U_{1}^{2}} \\
\operatorname{THD}_{i} & =\frac{\sum_{h=2}^{40} I_{h}^{2}}{I_{1}^{2}}
\end{aligned}
$$

where $U_{h}$ and $I_{h}$ denote the RMS values of voltage and current harmonic components of order $h$.

Let us evaluate the ratio $L F$, power factor $P F$, voltage and current THDs, and norms of current vectors for the ILs and CFLs connected as a single- and wye-connected three-phase loads.

\section{Results}

In the case study, the 4 wire cable with the wire impedance $Z=0.2474+j 0.0415 \Omega$ is applied to connect the load in the form of CLFs or ILs with the distribution transformer (150 kVA, 20/0.4 kV, Dyn5). Fig. 1 shows the supply voltage waveform and its amplitude spectrum at the load terminals.
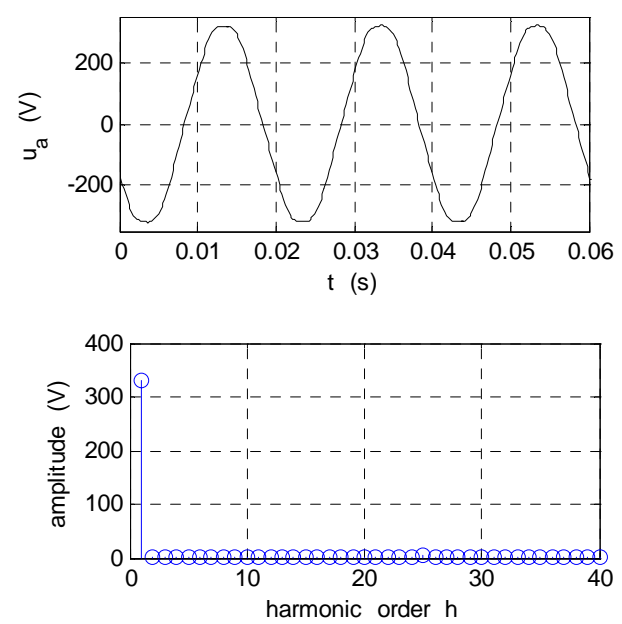

Figure 1: Supply voltages $u_{a}$ and its amplitude spectrum $\left(T H D_{u}=0.02\right)$

Fig. 2 shows the current waveform and its amplitude spectrum in the case of IL with the power $92.08 \mathrm{~W}$. It produces similar luminous flux as a CFL with the power $19.01 \mathrm{~W}$. The current waveform for the CFL is given in Fig. 3 together with its amplitude spectrum. Comparison of THDs for the IL and CFL shows that the current waveform is undistorted in the case of IL, where $T H D_{i}=0.02$, while it is substantially distorted in the case of CFL, where $T H D_{i}=0.97$.
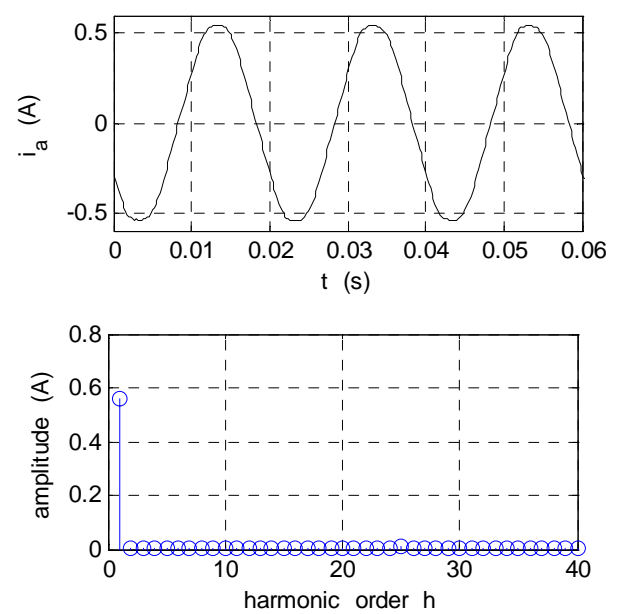

Figure 2: Current $i_{a}$ and its amplitude spectrum - IL $(\mathrm{THD}=0.02)$
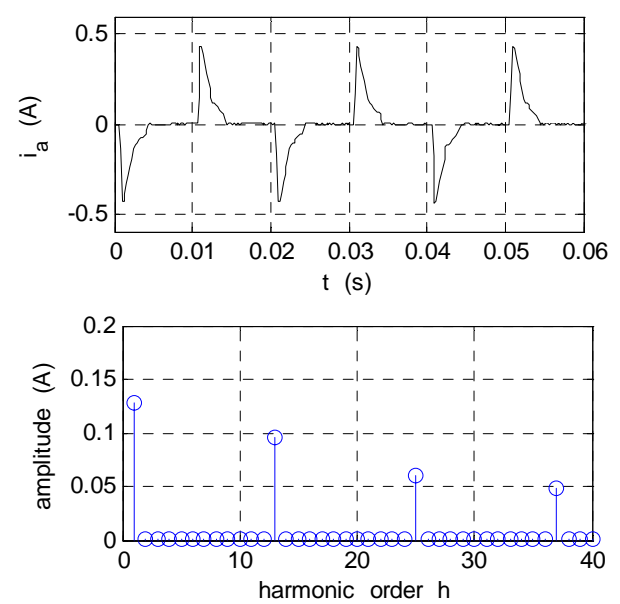

Figure 3: Current $i_{a}$ and its amplitude spectrum - CFL (THD=0.97)

In the case of a single-phase load, the current flows from the transformer's terminals through the line conductor, load and neutral conductor back to the transformer's terminals. Considering that the ohmic resistance of the line and neutral conductors equal the real part of the wire impedance $R=R_{n}=0.2474 \Omega$, the losses related with the energy transmission in the discussed cable between the transformer and the load can be calculated by (14) to (16), while the ratio $L F$ can be calculated by (17) or (18). The results are presented in Table I for the IL and CFL.

Table I: Load power $P$, energy transmission losses $P_{l}$, power factor $P F$ and ratio $L F=P_{l} / P$ for IL and CFL

\begin{tabular}{|l|l|l|l|l|}
\hline & $P(\mathrm{~W})$ & $P_{l}(\mathrm{~W})$ & $P F$ & $L F$ \\
\hline IL & 92.083 & 0.1560 & 1.00 & 0.0017 \\
\hline CFL & 19.009 & 0.0182 & 0.60 & 0.0010 \\
\hline
\end{tabular}

The results presented in Table I clearly show that the power $P$ and the transmission losses $P_{l}$ are much lower in the case of CFL, where the same or very similar luminous flux is generated by the IL and CFL. The other question that must be answer is what happens with the transmission losses with respect to the unit of load power or delivered energy? In order to perform the analysis, the voltage waveform shown in Fig. 1 was used, while the current waveforms in Figs. 2 and 3 were multiplied by 
the constant factors $P_{n} / P$, where $P$ is the active power from Table I while $P_{n}$ is the new active power. In this way, the different active powers were set by scaling measured currents. The described procedure simulates measurement of more CFLs or ILs connected in parallel at constant supply voltage. Figs. 4 to 7 show the transmission losses $P_{l}$, ratio $L F=P_{l} / P$ and norms or (RMS) values $\|\mathbf{i}\|$ and $\left\|\mathbf{i}_{u}\right\|$ in the discussed cable as functions of the load power $P$ for IL and CFL.

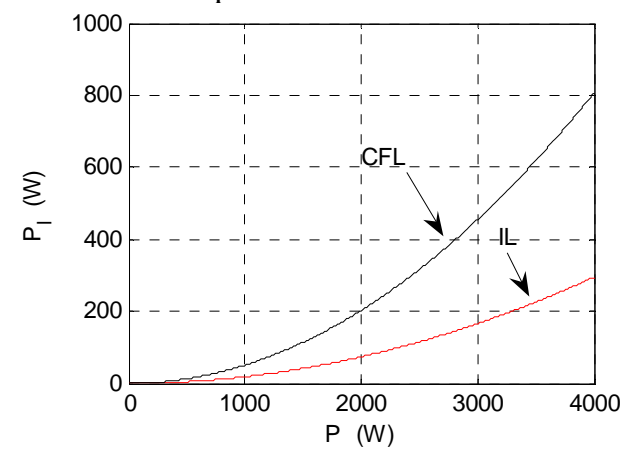

Figure 4: Transmission losses $P_{l}$ given as a function of load power $P$ for CFL and IL

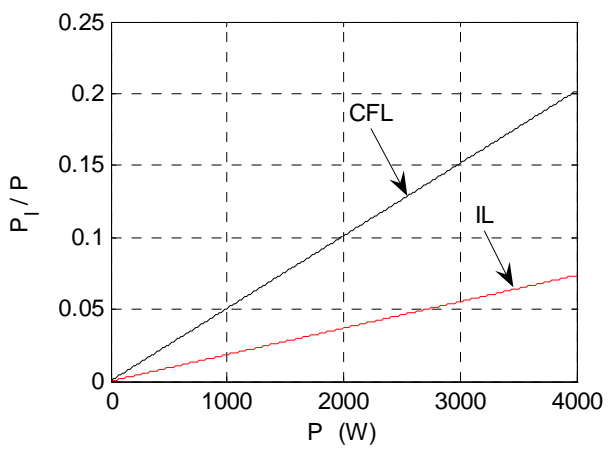

Figure 5: Ratio transmission losses / load power $L F=P_{l} / P$ given as function of load power $P$ for CFL and IL

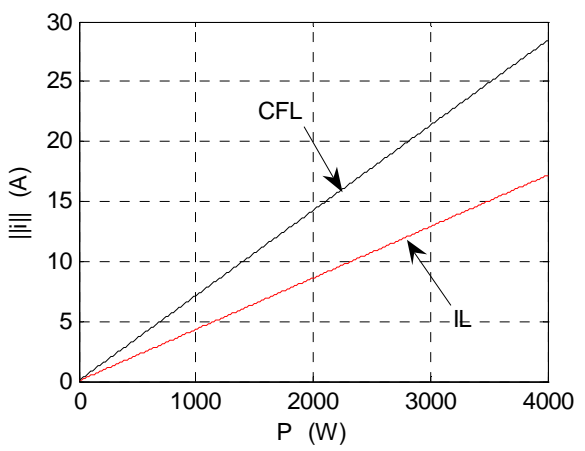

Figure 6: Current norm $\|\mathbf{i}\|$ (RMS value) given as function load power $P$ for CFL and IL

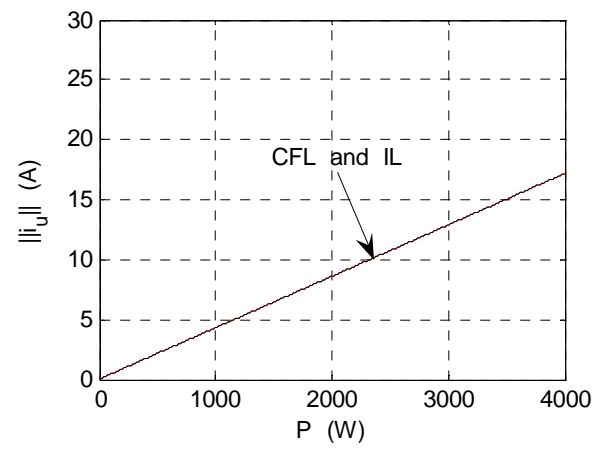

Figure 7: Current norm $\| \mathbf{i}_{u}$ given as function load power $P$ for CFL and IL
The results presented in Figs. 4 to 7 clearly show that the transmission losses cannot be reduced in the case of ILs. On the other hand, in the case of CFLs, the transmission losses are higher than they could be. The ratio between the actual transmission losses and transmission losses that cannot be avoided is proportional to the $1 / P F^{2}$. Thus, results presented in Figs. 4 to 7 clearly show that the losses related with the energy transmission with respect to the unit of load power are higher in the cases when CFLs are applied.

Let us analyze losses related with the energy transmission in a 4 wire system with neutral conductor in cases when the three-phase load is composed exclusively of wyeconnected ILs or CFLs. Fig. 8 shows the three-phase supply voltages $u_{a}, u_{b}$ and $u_{c}$. Figs. 9 and 10 show the line currents $i_{a}, i_{b}, i_{c}$ and the neutral conductor current $i_{n}$ in the case of wye-connected ILs, while Figs. 11 and 12 show the same variable in the case of wye-connected CFLs.

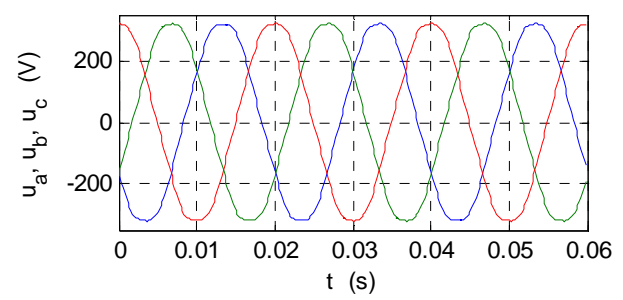

Figure 8: Three-phase supply voltages $u_{a}, u_{b}$ and $u_{c}$

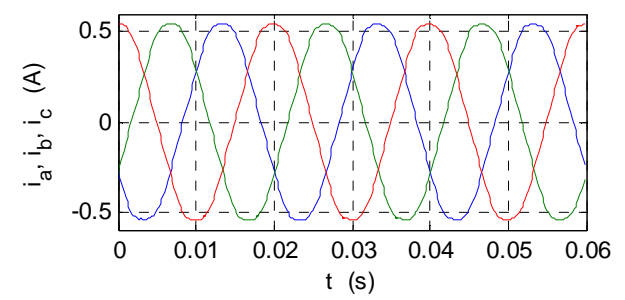

Figure 9: Wye-connected ILs: line currents $i_{a}, i_{b}$ and $i_{c}$

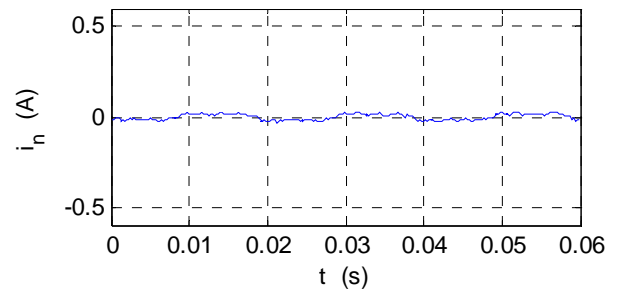

Figure 10: Wye-connected ILs: neutral conductor current $i_{n}$

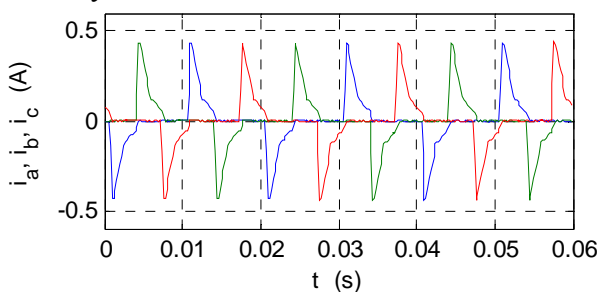

Figure 11: Wye-connected CFLs: line currents $i_{a}, i_{b}$ and $i_{c}$

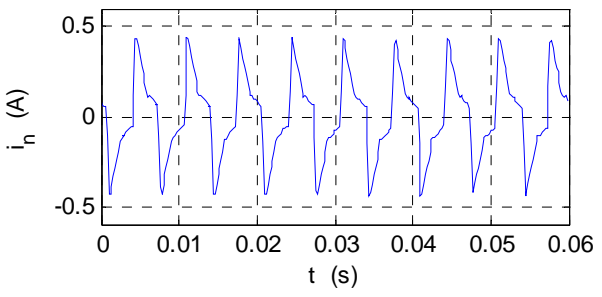

Figure 12: Wye-connected CFLs: neutral conductor current $i_{n}$ 
Let us present currents from Figs. 9 and 11 as a current vector i (1) and decompose it into its orthogonal components $\mathbf{i}_{\mathrm{u}}(5)$ and $\mathbf{i}_{\mathrm{uo}}(6)$, let us determine the neutral conductor current $i_{n}(11)$ and its components $i_{n u}(12)$ and $i_{\text {nио }}$ (13), due to $\mathbf{i}_{\mathrm{u}}$ and $\mathbf{i}_{\mathrm{uo}}$, and let us determine the ratio between the losses related with the energy transmission and the load power LF (17). Figs. 13 to 16 are given for the wye-connected ILs while Figs. 17 to 20 are given for the wye-connected CFLs. Figs. 13 and 17 show components of the vector $\mathbf{i}_{\mathrm{u}}$ in individual line currents. Similarly, Figs. 14 and 18 show components of the vector $\mathbf{i}_{\text {uо }}$ in individual line currents. The neutral conductor currents due to the $\mathbf{i}_{\mathrm{u}}$ are shown in Figs. 15 and 19 while the neutral conductor currents due to the $\mathbf{i}_{\text {uо }}$ are shown in Figs. 16 and 20.

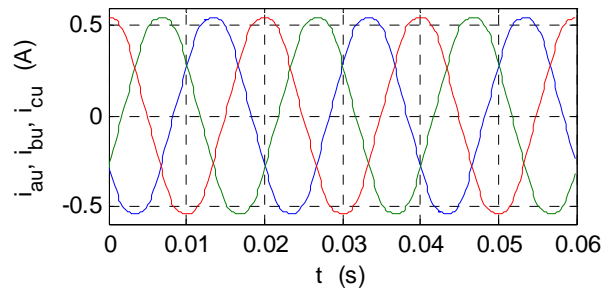

Figure 13: Wye-connected ILs: components of $\mathbf{i}_{u}$ in individual line currents

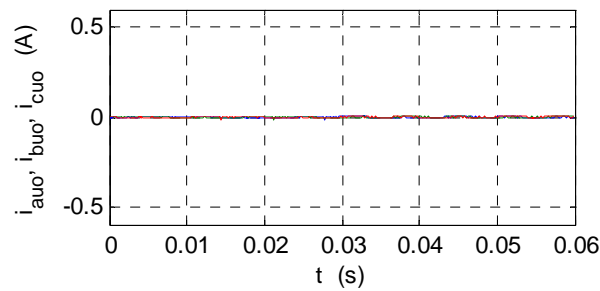

Figure 14: Wye-connected ILs: components of $\mathbf{i}_{u o}$ in individual line currents

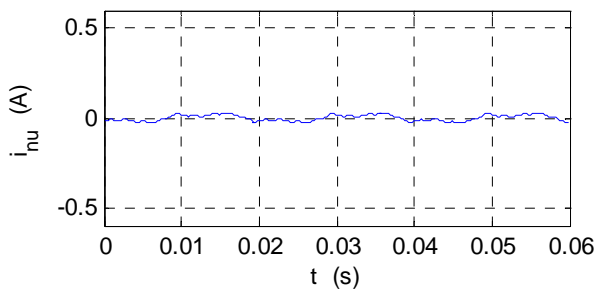

Figure 15: Wye-connected ILs: neutral conductor current due to $\mathbf{i}_{u}$

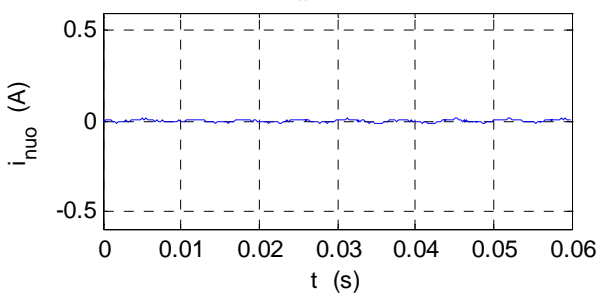

Figure 16: Wye-connected ILs: neutral conductor current due to $\mathbf{i}_{\text {uо }}$

The comparison of Figs. 9 and 13 shows that, in the case of ILs, the line currents are very close to the components of vector $\mathbf{i}_{u}$, which is the only indispensable for the energy transmission. On the contrary, the components of the vector $\mathbf{i}_{u o}$, which does not contribute to energy transmission represented in the form of active power, are small. They are shown in Fig. 14. The neutral conductor currents due to the current vectors $\mathbf{i}_{u}$ and $\mathbf{i}_{u о}$ are small. They are caused mostly by slightly unbalanced supply voltages.
In the case of CFLs, the comparison of the line currents, shown in Fig. 11, with components of the current vectors $\mathbf{i}_{\mathrm{u}}$ and $\mathbf{i}_{\mathrm{uo}}$ in individual line currents, shown in Figs. 17 and 18 , shows that only a minor part of the line currents (components of vector $\mathbf{i}_{\mathrm{u}}$ ) contributes to the energy transmission represented in the form of active power. Moreover, components of the vector $\mathbf{i}_{\mathrm{u}}$ in individual line currents give only a small contribution, which is shown in Fig. 19, to the total neutral conductor current, shown in Fig. 12. Major contribution to the neutral conductor current is shown in Fig. 20. It is caused by the current vector $\mathbf{i}_{\text {uo }}$ which does not contribute to the active power. Table II shows norms (RMS values) of signals shown in Figs. 9 to 20. They are calculated considering (3), (5), (6) and (11) to (13).

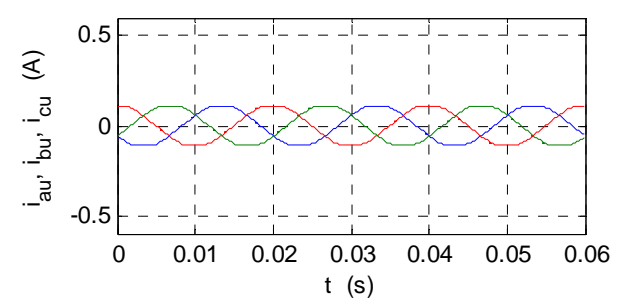

Figure 17: Wye-connected CFLs: components of $\mathbf{i}_{u}$ in individual line currents

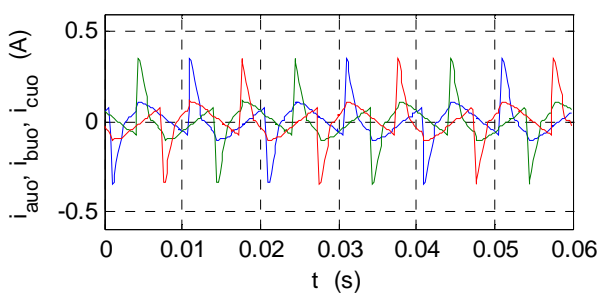

Figure 18: Wye-connected CFLs: components of $\mathbf{i}_{u o}$ in individual line currents

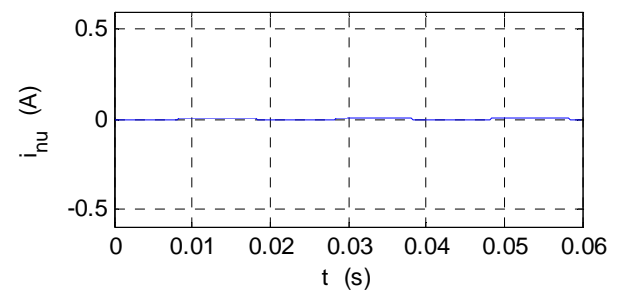

Figure 19: Wye-connected CFLs: neutral conductor current due

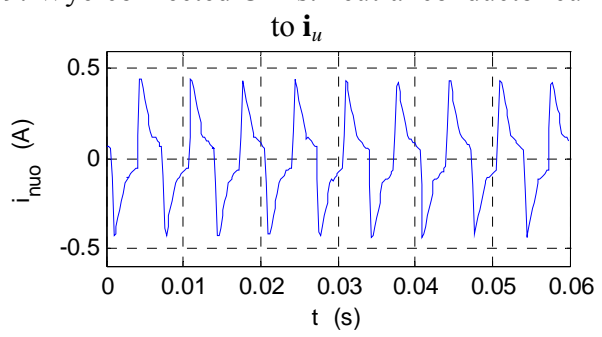

Figure 20: Wye-connected CFLs: neutral conductor current due to $\mathbf{i}_{\text {uo }}$

Table II: Norms (RMS values) of current vectors $\mathbf{i}, \mathbf{i}_{u}, \mathbf{i}_{u o}$ and neutral conductor currents $i_{n}, i_{n u}, i_{\text {nuo }}$

\begin{tabular}{|l|c|c|c|c|c|c|}
\hline & $\begin{array}{c}\|\mathbf{i}\| \\
\mathrm{A})\end{array}$ & $\begin{array}{c}\left\|\mathbf{i}_{u}\right\| \\
(\mathrm{A})\end{array}$ & $\begin{array}{c}\left\|\mathbf{i}_{\text {uо }}\right\| \\
(\mathrm{A})\end{array}$ & $\begin{array}{c}\left\|i_{n}\right\| \\
(\mathrm{A})\end{array}$ & $\begin{array}{c}\left\|i_{\text {nи }}\right\| \\
(\mathrm{A})\end{array}$ & $\begin{array}{c}\left\|i_{\text {nио }}\right\| \\
(\mathrm{A})\end{array}$ \\
\hline $\mathrm{CFL}$ & 0.2332 & 0.1406 & 0.1860 & 0.2304 & 0.0035 & 0.2313 \\
\hline $\mathrm{IL}$ & 0.6840 & 0.6840 & 0.0059 & 0.0165 & 0.0168 & 0.0071 \\
\hline
\end{tabular}


Table III: Load power $P$, energy transmission losses $P_{l}$, power factor $P F$ and ratio $L F=P_{l} / P$ for wye-connected ILs and CFLs

\begin{tabular}{|l|l|l|l|l|}
\hline & $P(\mathrm{~W})$ & $P_{l}(\mathrm{~W})$ & $P F$ & $L F$ \\
\hline IL & 276.2306 & 0.1158 & 1.00 & 0.00042 \\
\hline CFL & 56.7927 & 0.0266 & 0.60 & 0.00047 \\
\hline
\end{tabular}

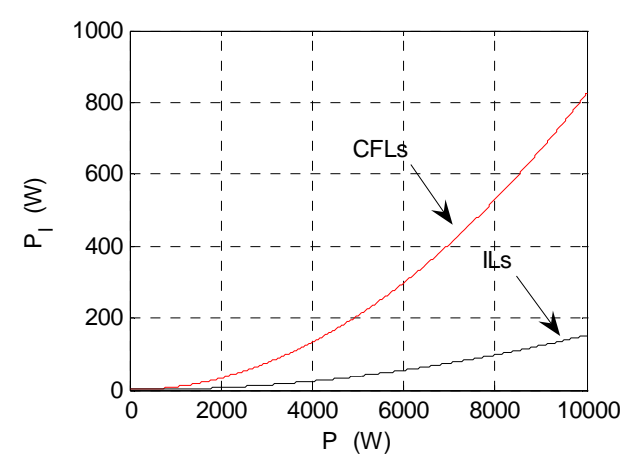

Figure 21: Transmission losses $P_{l}$ given as a function of load power $P$ for wye-connected CFLs and ILs

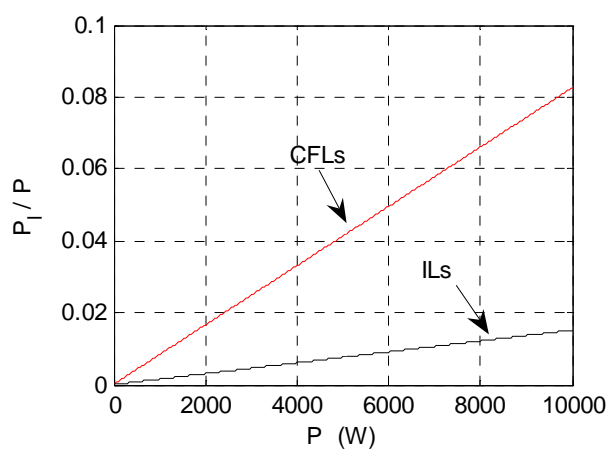

Figure 22: Ratio $L F=P_{l} / P$ given as function of load power $P$ for wye-connected CFLs and ILs

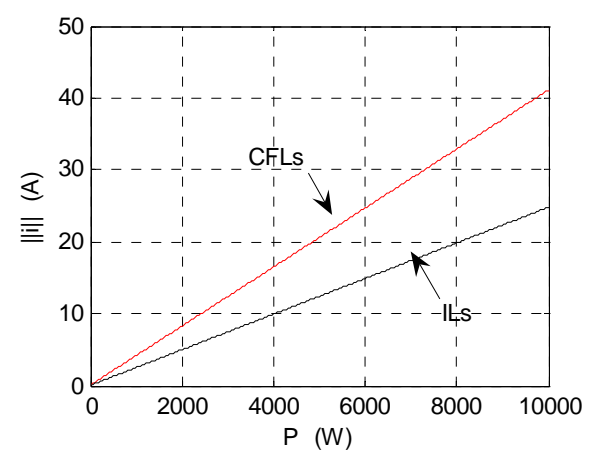

Figure 23: Current vector norm $\|\mathbf{i}\|$ given as function of load power $P$ for wye-connected CFLs and ILs

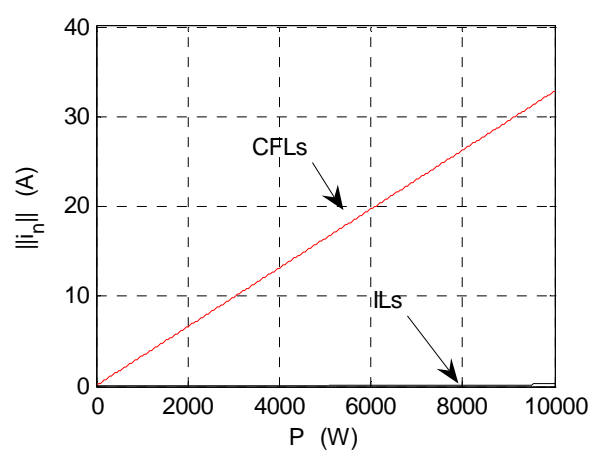

Figure 24: RMS value of neutral conductor current $\left\|i_{n}\right\|$ given as function of load power $P$ for wye-connected CFLs and ILs
Table III shows load power $P(2)$, losses related with the energy transmission $P_{l}(16)$, power factor $P F(9)$ and ratio $L F$ (17). They are given for currents and voltages shown in Figs. 8 to 12. The results presented in Table III show that the factor $L F$ (the ratio between the transmission losses $P_{l}$ and the load power $P$ ) is higher in the case of wye-connected CFLs, in spite of much higher load power in the case of wye-connected ILs.

Results presented in Figs. 21 to 24 show the transmission losses $P_{l}(16)$, the ratio $L F=P_{l} / P(17)$, the current vector norm $\|\mathbf{i}\|(3)$, and the (RMS) value of the neutral conductor current $\left\|i_{n}\right\|$. They are given as functions of increasing load power $P$ for wye-connected CFLs and ILs. Figs. 21 and 22 clearly show that CFLs cause higher transmission losses than ILs for the same load power. The norms (RMS values) $\|\mathbf{i}\|$ and $\left\|i_{n}\right\|$ shown in Figs. 23 and 24 are much higher in the case of CFLs. In the case of wye-connected CFLs, the load power is limited by the cross-section of the neutral conductor

\section{Conclusion}

The results presented in this contribution clearly show that CFLs require only 20 to 25 percents of the power required by ILs to produce similar luminous flux. There is no doubt that CFLs can save a lot of energy. However, with the increasing power of CFLs installed in distribution networks, the energy transmission related losses, with respect to the unit of load power or unit of deliver energy, must be considered as well. From this viewpoint, at least in the presented case study, the CFLs' performances are bed. The power factor of 0.6 is very low. Combinations of nonlinear loads, like CFLs, computes, UPSs and similar devices, can make it even lower, which can substantially increase transmission losses. However, in the distribution networks the nonlinear and linear loads are normally combined and unbalanced. They all must be considered in the analysis, in order to obtain relevant evaluation of transmission losses.

\section{References}

[1] H. Akagi, Y. Kanazawa in A. Nabae, "Instantaneous reactive power compensators comprising switching devices without energy storage components," IEEE Transactions on Industry Applications, vol. 20, no. 3, pp. 625-631, 1984

[2] J. L. Willems, "A new interpretation of the Akagi-Nabae power components for nonsinusoidal three-phase situations," IEEE Transactions on Instrumentation and Measurements, vol. 41, no. 4, pp. 523-527, 1992.

[3] L. S. Czarnecki, "What is wrong with the Budeanu concept of reactive and distortion power and why it should be abandoned," IEEE Transactions on Instrumentation and Measurements, vol. 36, no. 3, pp. 834-837, 1987.

[4] L. S. Czarnecki, "A time-domain approach to reactive current minimization in nonsinusoidal situations," IEEE Transactions on Instrumentation and Measurements, vol. 39, no. 5, pp. 698-703, 1990. 